\title{
A Shadow Touching Technique for Interactive Projector Devices
}

\author{
Lan-Rong Dung and Ren-Yu Huang \\ National Chiao Tung University, Hsinchu 30010, Taiwan \\ lennon@faculty.nctu.edu.tw
}

\begin{abstract}
Touch devices have become one of the major elements in today's most electronic products. Touch technology can be divided into different types as resistive, capacitive, infrared, and so on. However, as the amount of detection sensors increases, most types of touch screen suffer from more cost and lower yield when the accuracy of touch location or larger size is required. Therefore, it is essential to design a different kind of touch technology with accurate detection and the cost is not proportional to its size. This paper proposed a kind of touch system using the distance between the object (eg. finger or stylus) and its shadow to determine whether the object touched the screen or not, and locate the touch point. It can be applied to any interactive projection system without using a lot of touch detecting sensor. Therefore, no additional costs are required for a large touch screen. The proposed touch system only requires one camera and one IR source, and cooperates with an interactive projector to detect the occurrence of touching and its location. The proposed system achieves an average detection rate of $97.53 \%$ if the tolerance of detected touch coordinates is in \pm 10 pixels.
\end{abstract}

Keywords: Shadow touch, touch screen, interactive projector.

\section{Introduction}

Panels are quite popular due to its instinctive input method as a human-machine interface. There are three units in this kind of system. Most of them have sensors and coordinates calculation units, and some have the special signal transmission unit. The sensor unit is used to receive signal and generate information that can define the coordinates. The coordinates calculation unit transforms the information generated by sensor unit to coordinates or some other signals that can determine the location. There are some special touch system such as electromagnetic touch system must have additional transmission unit to generating specific signals for sensor unit.

The most common materials covered on the intermediate or small-sized touch panel are resistors and capacitors. However, as the panel size increases, the number of sensor units increases and leads to higher cost and lower yield rate. Therefore, these types of touch systems are not suitable for large-sized touch screen. On the contrary, ultrasonic, optical, electromagnetic, infrared, and vision-based

C. Stephanidis (Ed.): Posters, Part I, HCII 2013, CCIS 373, pp. 308-312, 2013.

(C) Springer-Verlag Berlin Heidelberg 2013 
touch system are more feasible to make a large or even extra large touch system since the sensor units will not increase with the panel size. However, these touch systems still have some drawbacks. For example, the cost of ultrasonic based touch system is relatively expensive. The electromagnetic based touch system requires an electromagnetic pen. The infrared and vision based system need more than one camera to locate the position.

This paper focused on developing a vision-based touch system with large-sized screen. The vision-based touch system is quite suitable for large touch screen. It has a camera that can catch images to calculate the location and decide the event of touching. There are many similar techniques of vision-based touch systems proposed by other researches [1-5. The main issue of these touch systems is to determine whether the action of object really "touched" the screen or just a movement 2].

This paper proposed a vision-based touch technique that use the shadow information to detect the touch event and location. This system uses only one camera and does not use the gesture recognition to distinguish the touch event and movment. Hence it is more instinctive, user-friendly, and save more cost relative to two camera system.

\section{Proposed System}

The proposed touch system is shown in Fig. 1. The touch system includes an interactive projector with an infrared camera, an IR filter, a projection screen and an infrared light source. The interactive projector projects contents on projection screen, and the camera capture user's action in front of projection screen. The IR filter is installed in front of the camera lens to filter out interferences from the projection contents and other visible light. The infrared light source supply illumination to the system. The images captured by camera will be processed. Therefore, when the user touches projection screen, the system will detect the touches, and estimating the touching location.

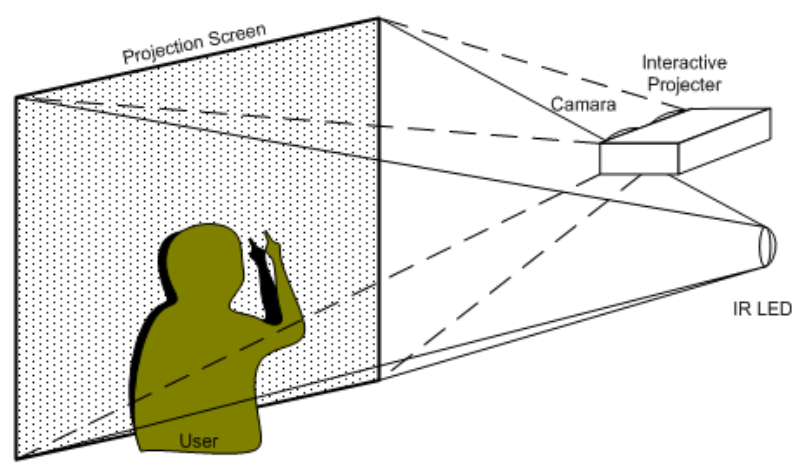

Fig. 1. Proposed System Framework 


\subsection{Shadow and Object Bi-level Images}

After noise reduction and illumination correction, we can seperate the shadow pixels by using the threshold estimated in the initializaiton step. The preliminary bi-level image of shadow can be obtained by the following equation:

$$
S(x, y)=\left\{\begin{array}{l}
1, \text { if } f(x, y)<\theta_{s}(x, y) . \\
0, \text { otherwise. }
\end{array}\right.
$$

Before segmenting the object from image, we first subtract current frame from the background image. Since the difference of two images in the same scene contains real object or noise. Hence we have to set a condition to reduce the noise effect. If the pixel absolute difference is greater than some pre-defined constant $T_{f}$, then set the pixel as the "foreground" pixel. This makes the system robust to segment the object from image. In our experiments, $T_{f}=9$ is enough to distinguish the difference of object from difference of noise. The bi-level image of object can be obtained by:

$$
O(x, y)=\left\{\begin{array}{c}
1, \text { if } f(x, y)>\theta_{o}(x, y) \text { and } \\
|f(x, y)-b g(x, y)|>T_{f} \\
0, \text { otherwise. }
\end{array}\right.
$$

\subsection{Tips Detection}

After extracting the bi-level images of object and shadow, we have to find the possible locations of fingertip or stylus tip. We detect the tips of object and shadow by checking the four boundary of a 16x16 scanning mask, as shown in Fig. 2. If there is only one boundary contains the object or shadow pixels, then the tip is detected; otherwise, there is no tip in the mask. When the tip is detected, we calculate height and width of the tip. If the height is large enough and the width is small enough, then calculate and record the direction and coordinates of the tip.

\subsection{Touch Judgement}

After detecting the tips of object and shadow, we use the information of tips to determine touch-timing and touch-point. We trace the tips for more than one frame, and use four parameters to judge whether the object's tip touches the projection screen or not. The four parameters are direction of tips, the distance between object tip and shadow tip, frame counts which is the number of frames that include the same tip, and the approaching velocity of object's tip and shadow's tip. If the object's tip and shadow's tip are in the same direction and close enough, the the object's tip might be the peak of finger or stylus. If two successive frames have the same close object's tip and shadow's tip, the frames counts will increase by one. Then we calculate the approaching velocity of the object's tip and shadow's tip, if the object's tip and shadow's tip are getting closer in two successive frames, the approaching distance is added by the 


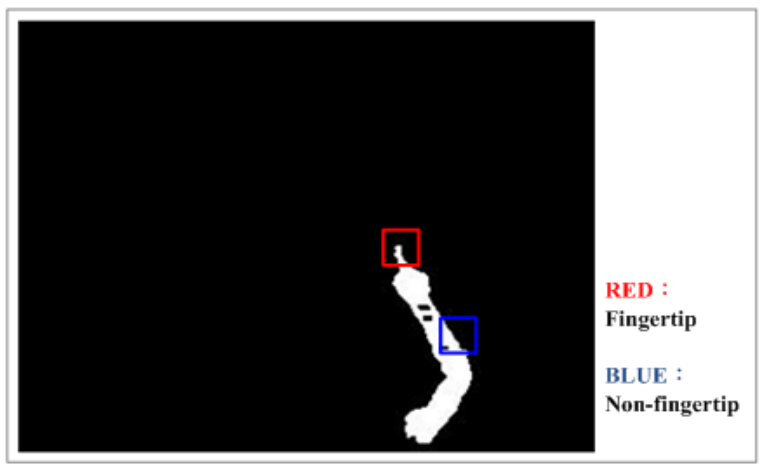

Fig. 2. Tip detection

Table 1. Detection rate and False Alarm Ratio

\begin{tabular}{|c|c|c|c|c|c|}
\hline $\begin{array}{c}\text { Test } \\
\text { Pattern }\end{array}$ & $\begin{array}{c}\text { Touch } \\
\text { Number }\end{array}$ & Hits & $\begin{array}{c}\text { Detection } \\
\text { Rate } \\
\text { (DR) }\end{array}$ & $\begin{array}{c}\text { False } \\
\text { Alarms }\end{array}$ & $\begin{array}{c}\text { False } \\
\text { Alarm } \\
\text { Ratio } \\
\text { (FAR) }\end{array}$ \\
\hline 1 & 81 & 81 & $100.00 \%$ & 0 & $0 \%$ \\
2 & 122 & 119 & $95.90 \%$ & 0 & $0 \%$ \\
3 & 51 & 49 & $96.08 \%$ & 0 & $0 \%$ \\
4 & 72 & 72 & $100.00 \%$ & 0 & $0 \%$ \\
5 & 104 & 101 & $97.16 \%$ & 0 & $0 \%$ \\
6 & 83 & 79 & $95.18 \%$ & 0 & $0 \%$ \\
7 & 87 & 84 & $96.55 \%$ & 0 & $0 \%$ \\
8 & 58 & 58 & $100.00 \%$ & 0 & $0 \%$ \\
9 & 59 & 59 & $100.00 \%$ & 0 & $0 \%$ \\
10 & 61 & 58 & $95.08 \%$ & 0 & $0 \%$ \\
11 & 73 & 70 & $95.89 \%$ & 0 & $0 \%$ \\
\hline Total & 851 & 830 & $97.53 \%$ & 0 & $0 \%$ \\
\hline
\end{tabular}

difference of the distance between object's tip and shadow's tip in two frames. Approaching velocity is approaching distance divided by frames counts, if the object's tip and the shadow's tip are getting closer in these successive frames, the approaching velocity will be positive. The formula for calculating approaching velocity is shown as Eq. (3).

$$
\begin{gathered}
V_{\text {approach }}(k)=\frac{D_{\text {approach }}(k)}{\text { Frame Counts }} \\
D_{\text {approach }}(k)=D_{\text {approach }}(k-1)+\Delta D \\
\Delta D=D(k)-D(k-1)
\end{gathered}
$$

Where $D(k)$ is the Euclidean distance between object's tip and shadow's tip in the k-th frame, $D_{\text {approach }}(k)$ is the approaching distance, and $V_{\text {approach }}(k)$ is the approaching velocity. 


\section{Conclusion}

This paper proposed an touch system which takes advantages of the relative relationship between finger or stylus and its shadow to determine whether a finger or stylus touches the screen. The touch system proposed in this paper needs only one camera and one IR source, and cooperate with an interactive projector to detect the occurrence of touches and locations. The proposed system does not using any touch detecting elements, hence no additional costs are needed for a greater touch screen. Comparing to other large-size touch panels, the proposed touch system is relatively low cost and portable.

We use 11 test sets to evaluate the performance of the proposed touch system. The test patterns are videos with 15 frames per second, and the frame size is $320 \times 240$ pixels. The performance of the proposed method is shown as Table 1 The proposed system achieves an average detection rate(DR) of $97.53 \%$ and false alarm ratio(FAR) of $0 \%$ if the tolerance of detected touch coordinate is in the range of \pm 10 pixels.

\section{References}

1. Mo, Z., Lewis, J.P., Neumann, U.: Smart Canvas: A Gesture- Driven Intelligent Drawing Desk System. In: 5th ACM symposium on Intelligent User Interfaces, pp. 239-243 (2005)

2. Bretzner, L., Laptev, I., Lindeberg, T.: Hand gesture recognition using multi-scale colour features, hierarchical models and particle filtering. In: Face and Gesture, pp. $423-428$ (2002)

3. Chen, X., Koike, H., Nakanishi, Y., Oka, K., Sato, Y.: Two-handed drawing on augmented desk system. In: Working Conference on Advanced Visual Interfaces (May 2002)

4. Hall, D., Gal, C.L., Martin, J., Chomat, O., Crowley, J.L.: Magicboard: a contribution to an intelligent office environment. the International Symposium on Intelligent Robotic Systems 35(3), 215-223 (1999)

5. Wellner, P.: Interacting with paper on the digital desk. ACM Trans. on Communications 36(7), 86-97 (1993)

6. Hardenberg, C.: Fingertracking and Handposture Recognition for Real-Time Human-Computer Interaction, http://iihm.imag.fr/hardenbe

7. Tasdizen, T., Jurrus, E., Whitaker, R.T.: Non-uniform illumination correction in transmission electron microscopy. In: MICCAI Workshop on Microscopic Image Analysis with Applications in Biology (September 2008)

8. Otsu, N.: A Threshold Selection Method from Gray Level Histogram. IEEE Trans. on Systems, Man and Cybernetics 9, 62-66 (1979)

9. Wu, K., Otoo, E., Suzuki, K.: Optimizing two-pass connected-component labeling algorithms. Pattern Analysis \& Applications 12(2), 117-135 (2009)

10. Barnes, L.R., Schultz, D.M., Gruntfest, E.C., Hayden, M.H., Benight, C.C.: Corrigendum: False alarm rate or false alarm ratio? Weather Forecasting (2009)

11. Gonzalez, R.C., Woods, R.E.: Digital Image Processing, 3rd edn. Prentice Hall (2008) 